\title{
$\mathrm{BMJ}$
}

\section{Use of venlafaxine compared with other antidepressants and the risk of sudden cardiac death or near death: a nested case-control study}

\author{
Carlos Martinez, adjunct professor, ${ }^{1}$ consultant epidemiologist, ${ }^{2}$ Themistocles L Assimes, assistant professor \\ of medicine, ${ }^{3}$ Daniel Mines, pharmacoepidemiologist, ${ }^{4}$ Sophie Dell'Aniello, research assistant, ${ }^{1}$ Samy Suissa, \\ professor and director
}

\section{${ }^{1}$ Centre for Clinical Epidemiology, Jewish General Hospital, and Departments of Epidemiology and Biostatistics and of Medicine, McGill University, Montreal, Canada ${ }^{2}$ Carlos Martinez, Frankfurt, Germany \\ ${ }^{3}$ Division of Cardiovascular Medicine, Stanford University School of Medicine, Stanford, CA, USA \\ ${ }^{4}$ Wyeth Research, Philadelphia, USA}

Correspondence to: S Suissa, Centre for Clinical Epidemiology, Jewish General Hospital, 3755 Cote Ste-Catherine, Suite H4, Montreal, Québec, Canada H3T 1E2 samy.suissa@mcgill.ca

Cite this as: BMJ 2010;340:c249 doi:10.1136/bmi.c249

\section{ABSTRACT}

Objective To assess whether use of the antidepressant venlafaxine is associated with an increased risk of sudden cardiac death or near death compared with other commonly used antidepressants.

Design Population based observational study.

Setting We did a nested case-control analysis within a new user cohort formed using the United Kingdom General Practice Research Database.

Participants New users of venlafaxine, fluoxetine, citalopram, or dosulepin on or after 1 January 1995, aged 18 to 89 years, with a diagnosis of depression or anxiety. Participants were followed-up until February 2005, or the occurrence of sudden cardiac death or near death, identified from medical records indicating non-fatal acute ventricular tachyarrhythmia, sudden death due to cardiac causes, or out of hospital deaths from acute ischaemic cardiac events. For each case, 30 controls were selected matched for age, sex, calendar time, and indication. We used conditional logistic regression to calculate the adjusted odds ratio of sudden cardiac death or near death associated with current use of venlafaxine compared with current use of fluoxetine, citalopram or dosulepin. Results 207384 participants were followed-up for an average of 3.3 years. There were 568 cases of sudden cardiac death or near death, which were matched to 14812 controls. The adjusted odds ratio of sudden cardiac death or near death associated with venlafaxine use was 0.66 (95\% confidence interval 0.38 to 1.14 ) relative to fluoxetine use, whereas compared with citalopram it was 0.89 (0.50 to 1.60) and with dosulepin 0.83 (0.46 to 1.52 ).

Conclusions In this large, population based study, the use of venlafaxine was not associated with an excess risk of sudden cardiac death or near death compared with fluoxetine, dosulepin, or citalopram, in patients with depression or anxiety.

\section{INTRODUCTION}

The safety of antidepressant drugs, particularly the newer agents, has been the subject of much debate. The selective serotonin receptor inhibitors (SSRI), as well as more recent agents such as venlafaxine, a serotonin-norepinephrine reuptake inhibitor (SNRI), have received special scrutiny from regulators. Although the greatest attention has focused on the suicide associated risks with these agents, three observational studies conducted in the United Kingdom reported a higher rate of fatal overdose with venlafaxine use compared with SSRIs. ${ }^{1-3}$ While these studies suggested that venlafaxine is more toxic in overdose, their design could not measure or adjust for patient factors that could also account for this observation, if the drugs were selectively prescribed to patients with different underlying risks of suicide. ${ }^{45}$

The mechanism of death in patients who died from venlafaxine overdose has not been well established. One hypothesis is that venlafaxine promotes haemodynamically significant (herein referred to as malignant) ventricular tachyarrhythmias through ion channel effects that prolong the QRS and/or QT interval. One in vitro study found that venlafaxine inhibited the fast sodium channel in guinea pig myocytes, but the study was not conducted under physiological conditions. ${ }^{6}$ In vivo, QRS prolongation has been reported in the overdose setting but not seen in randomised controlled trials. ${ }^{78}$ Clinically important increases in the QTc interval were observed rarely during registration trials, ${ }^{9}$ in uncontrolled prospective cohort studies, ${ }^{10}$ and in the overdose setting. ${ }^{11}{ }^{12}$ No cases of malignant ventricular arrhythmias were reported in the pre-registration clinical trials of venlafaxine, ${ }^{8}$ but the studies were not powered to detect such rare adverse events. Another possible mechanism through which venlafaxine could promote arrhythmias is by precipitating cardiac ischaemia, given that the drug can increase blood pressure and heart rate..$^{101314}$

Concerns that venlafaxine was less safe than SSRIs in part due to cardiotoxicity led to regulatory action. In December 2004 the UK Medicines and Healthcare products Regulatory Agency (MHRA) restricted prescription of venlafaxine to specialists and contraindicated its use in patients with heart disease, electrolyte 
imbalance, or in patients who are hypertensive. ${ }^{1516}$ After further review, in May 2006 the MHRA revised its regulatory position. The new prescribing information again allowed prescribing by non-specialists (except at very high doses) and updated the cardiac contraindications, advising now that only patients at very high risk of ventricular arrhythmia or with uncontrolled hypertension should not use venlafaxine. ${ }^{917}$ However, we know of no systematic study that has assessed the risk of malignant arrhythmias or sudden cardiac death associated with the use of venlafaxine in usual clinical practice.

We therefore used a population based observational approach to assess the risk of out-of-hospital haemodynamically significant acute ventricular tachyarrhythmia or sudden cardiac death associated with venlafaxine use relative to the use of fluoxetine, citalopram, or dosulepin in patients treated for depression or anxiety.

\section{METHODS}

We did a cohort study with a nested case-control analysis using data obtained from the United Kingdom

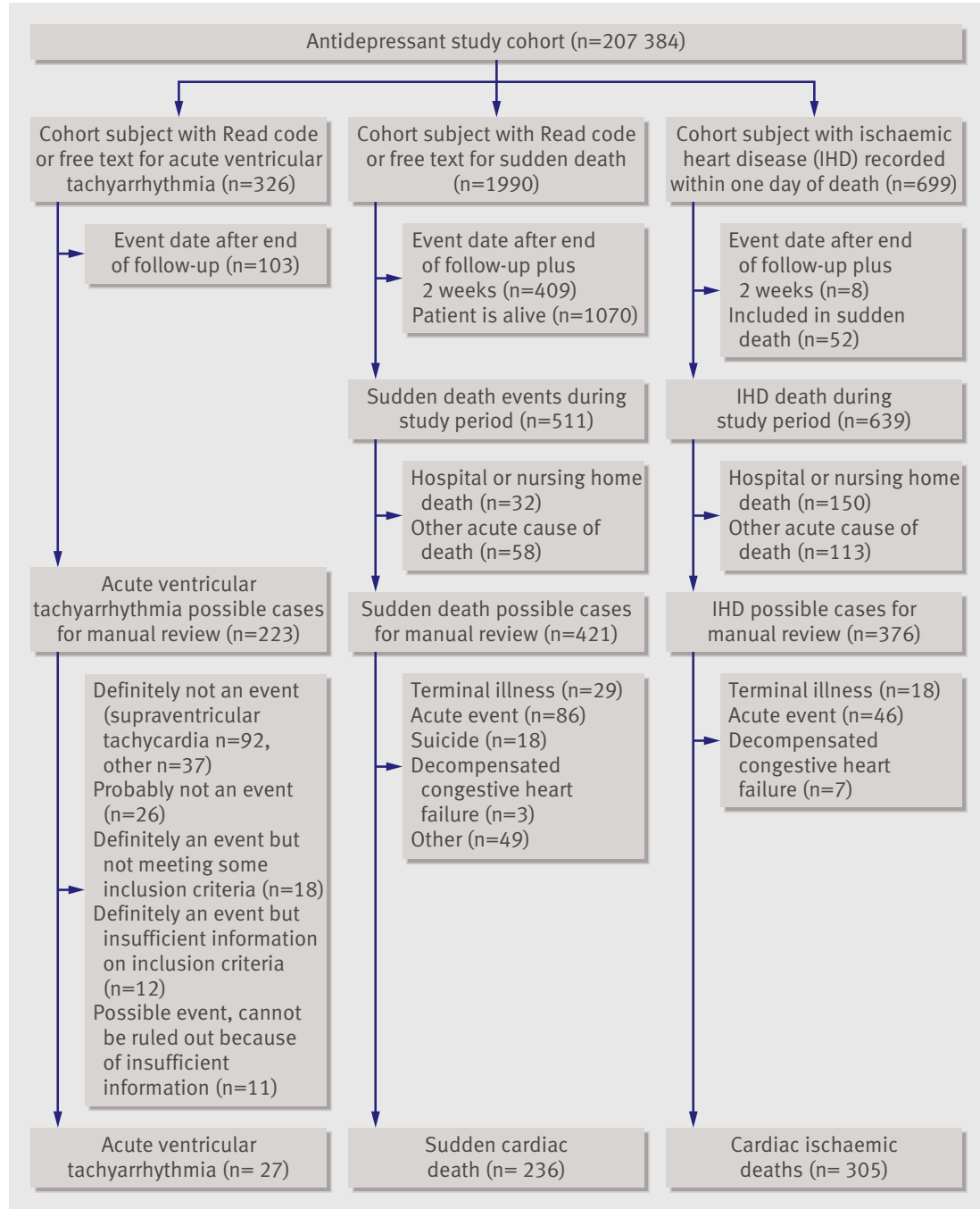

Flowchart of case ascertainment
General Practice Research Database (GPRD). This database contains more than 35 million person years of data from patient records continuously collected since 1987. Participating general practices currently contribute data on more than 3 million patients, roughly $5 \%$ of the UK population. Data collected included demographics, medical diagnoses, all prescriptions, referrals to secondary care, and hospital discharge reports. Participating general practitioners (GPs) receive training in data entry, and once practices have met certain quality standards, they are deemed as "up-to-standard." 18 Diagnoses are recorded using OXMIS or Read codes.

\section{Study cohort and design}

The study cohort has previously been used to assess the risk of suicide in patients treated with venlafaxine. ${ }^{19}$ Briefly, it was formed from all new users of venlafaxine, fluoxetine, citalopram, or dosulepin on or after 1 January 1995. We defined new users as patients who had received no prescription of the study drug in the year before cohort entry. Patients were aged between 18 and 89 years on the date of the incident prescription, and only patients with a clinical record for depression or anxiety on the date of or at any time before the incident prescription were selected. Patients were included in the cohort if they had a permanent registration status with a participating general practice, had at least a one year longitudinal record before the incident prescription, had an acceptable patient status for data quality, and originated from a general practice which was up to standard for at least a year before the incident prescription. ${ }^{20}$ Patients with a history of life threatening ventricular tachyarrhythmia, cardioversion, aborted cardiac arrest, or implantation of a cardiac defibrillator before cohort entry were excluded. Patients with a congenital conduction disorder or advanced cardiomyopathy (hypertrophic or dilated) before cohort entry or at any time during follow-up were also excluded.

Patients were followed-up over time from their incident prescription date (cohort entry) until the earliest of occurrence of the study outcome, death, transfer out of practice, or the practice's last collection date before February 2005, when data for this study were extracted. We used a case-control analysis nested within the cohort to deal with the complex time-varying nature of antidepressant use. ${ }^{2122}$

\section{Case definition}

The unifying feature of our composite outcome of sudden cardiac death or near death was the occurrence of a malignant ventricular tachyarrhythmia, which typically occurs in the presence of coronary atherosclerosis, its complications, or other structural heart disease. ${ }^{23}$ For near deaths, the occurrence of such a tachyarrhythmia was well documented and supported by the report of symptoms of presyncope or syncope before or during the event and/or the necessity of emergent cardioversion. For individuals who died, the tachyarrhythmia was often presumed because 
Table 1|Characteristics of cases and controls in the year before index date. Data are frequencies (percentages) unless otherwise stated. Percentages cannot be calculated directly from the corresponding frequencies as they are weighted by the number of controls matched to each case.

\begin{tabular}{|c|c|c|}
\hline & $\begin{array}{c}\text { Cases } \\
(n=568)\end{array}$ & $\begin{array}{c}\text { Controls } \\
(n=14812)\end{array}$ \\
\hline Age at index date, mean (SD)* & $72.9(12.7)$ & $72.9(12.8)$ \\
\hline Men $(\%)^{*}$ & $258(45.4)$ & $6404(45.4)$ \\
\hline \multicolumn{3}{|l|}{$\begin{array}{l}\text { Indication for cohort entry } \\
\text { antidepressant* }\end{array}$} \\
\hline Depression & $455(80.1)$ & $12893(80.1)$ \\
\hline Anxiety & $87(15.3)$ & $1594(15.3)$ \\
\hline Both & $26(4.6)$ & $325(4.6)$ \\
\hline \multicolumn{3}{|l|}{ Obesity status } \\
\hline Obese (BMI $\geq 30)$ & $86(15.1)$ & $2093(13.4)$ \\
\hline Non-obese $(\mathrm{BMI}<30)$ & $372(65.5)$ & $10818(73.3)$ \\
\hline Missing information & $110(19.4)$ & 1901 (13.3) \\
\hline \multicolumn{3}{|l|}{ Smoking status } \\
\hline Smoker & $249(43.8)$ & $5565(37.3)$ \\
\hline Non-smoker & $239(42.1)$ & $7429(50.3)$ \\
\hline Missing information & $80(14.1)$ & $1818(12.5)$ \\
\hline Alcohol abuse & $16(2.8)$ & $139(1.0)$ \\
\hline \multicolumn{3}{|l|}{ Depression/anxiety } \\
\hline Depression & $135(23.8)$ & $3751(23.8)$ \\
\hline Severe & $11(1.9)$ & $333(2.1)$ \\
\hline Moderate & $39(6.9)$ & $1372(8.7)$ \\
\hline Mild & $85(15.0)$ & $2046(13.0)$ \\
\hline Anxiety & $27(4.8)$ & $668(5.3)$ \\
\hline Both & $35(6.2)$ & $771(5.2)$ \\
\hline None & $371(65.3)$ & $9622(65.7)$ \\
\hline Suicide attempt & $5(0.9)$ & $59(0.4)$ \\
\hline
\end{tabular}

most deaths were not witnessed. Accordingly, three sources of out of hospital cases were identified: patients with non-fatal but haemodynamically significant acute ventricular tachyarrhythmia; patients with a record of sudden death due to cardiac causes ischaemic or otherwise; and patients seeming to have died out of hospital from an acute ischaemic cardiac event that was not necessarily labelled as sudden.

First, potential cases of acute ventricular tachyarrhythmia were identified from Read or OXMIS codes and word strings in the free text comments of GPs, in which case the complete de-identified free text was obtained from the GPRD. This text was reviewed by one of the authors (CM), blinded to exposure status, to assess whether additional chart information should be requested from the GP to clarify if the outcome of interest had occurred. The complete clinical profile of all these potential acute ventricular tachyarrhythmia cases was independently reviewed by two authors (CM and TA), who classified them as definite or not, and any discordance was resolved by consensus after further review and discussion. Then, sudden cardiac death cases were identified from Read/OXMIS code for "sudden death" or a word string in a free text comment that suggested the occurrence of a sudden death. Death certificates were obtained from the Office for National Statistics for England and Wales or the General Register Office for Scotland and Northern Ireland for the subset of those individuals who did not have a Read/OXMIS code or a free text comment that clearly stated a cause of death. Finally, people dying in the community from an acute cardiac ischaemic event that was not necessarily labelled as sudden formed the third group of potential cases, identified by scanning the Read/OXMIS codes for a code of ischaemic heart disease or myocardial infarction on the date of death (plus or minus one day). The complete profile of all potential sudden cardiac deaths and acute cardiac ischaemic deaths was subjected to a computer algorithm that identified and excluded deaths occurring either in hospital or in nursing homes and deaths apparently due to non-cardiac pathology. This algorithm was optimised after comparing the results of the initial run of the algorithm with the results of a manual review of a random set of 50 potential sudden death cases by two authors (CM and TA). All potential cases not excluded by the optimised computer algorithm then underwent a manual review by one of the authors $(\mathrm{CM})$ to further exclude cases that did not meet all inclusion and exclusion criteria.

The definite acute ventricular tachyarrhythmia, sudden cardiac death, and acute ischaemic cardiac death cases were combined to form the series of cases of sudden cardiac death or near death. The date of occurrence was designated the index date of the case.

\section{Control selection}

For each case we randomly selected up to 30 controls from the cohort. These controls were matched on cohort entry date (date of incident prescription plus or minus 6 months), year of birth, sex, and indication (depression and/or anxiety). Additionally, controls had to be at risk of the event on the case's index date. Thus, the control's index date was defined as the date that corresponded to the same follow-up time as its matched case. When fewer than 30 controls were available for a given case, we relaxed the matching criteria for cohort entry to within one year. If after this step, a given case had fewer than 30 controls, all available individuals were used.

\section{Antidepressant drug exposure}

For each case and their matched controls, we extracted all prescription records for the study drugs and all other antidepressants before the index date. We calculated the duration of each prescription, starting with the last prescription prior to index date, from the number of tablets prescribed combined with the dosing instructions. When we were unable to derive duration of exposure from the available information we assumed a prescription length of 28 days. For unusually low or high values of prescription length, we assigned a minimum of seven days and a maximum of 90 days to the prescription length. These assumptions are based on current prescribing practices in the UK.

We defined current exposure to treatment by a prescription whose duration included the index date. As 
Table 2|Cardiovascular comorbidity of cases and controls before index date. Data are frequencies (percentages) unless otherwise stated. Percentages cannot be calculated directly from the corresponding frequencies as they are weighted by the number of controls matched to each case.

\begin{tabular}{|c|c|c|}
\hline & $\begin{array}{l}\text { Cases } \\
(n=568)\end{array}$ & $\begin{array}{c}\text { Controls } \\
(n=14812)\end{array}$ \\
\hline \multicolumn{3}{|l|}{ In 2 years before index date } \\
\hline \multicolumn{3}{|l|}{$\begin{array}{l}\text { Acute myocardial infarction before } \\
\text { index date (\%) }\end{array}$} \\
\hline $0-3$ months & $14(2.5)$ & $28(0.2)$ \\
\hline 3-6 months & $7(1.2)$ & $27(0.2)$ \\
\hline 6-12 months & $10(1.8)$ & $57(0.4)$ \\
\hline $12-15$ months & $6(1.1)$ & $29(0.2)$ \\
\hline $15-18$ months & $1(0.2)$ & $28(0.2)$ \\
\hline $18-24$ months & $2(0.4)$ & $62(0.5)$ \\
\hline \multicolumn{3}{|c|}{ In the year before the year before index date } \\
\hline Cardiac revascularisation & $1(0.2)$ & $47(0.3)$ \\
\hline Intraventricular conduction delay & $0(0.0)$ & $0(0.0)$ \\
\hline Supraventricular arrhythmia & $29(5.1)$ & $234(1.6)$ \\
\hline Left ventricular hypertrophy & $2(0.4)$ & $8(0.1)$ \\
\hline Coronary artery disease and angina & $39(6.9)$ & $628(4.3)$ \\
\hline Congestive heart failure (CHF) & $51(9.0)$ & $368(2.5)$ \\
\hline Severe $\mathrm{CHF}^{\star}$ & $37(6.5)$ & $256(1.7)$ \\
\hline Ischaemic stroke & $9(1.6)$ & $199(1.5)$ \\
\hline Transient ischaemic attack & $6(1.1)$ & $204(1.5)$ \\
\hline Diabetes & $78(13.7)$ & $791(5.0)$ \\
\hline Peripheral vascular disease & $11(1.9)$ & $166(1.2)$ \\
\hline Hyperlipidaemia & $7(1.2)$ & $198(1.4)$ \\
\hline Hypertension & $38(6.7)$ & $799(5.4)$ \\
\hline Hypokalaemia & $4(0.7)$ & $39(0.3)$ \\
\hline Hypomagnesaemia & $0(0.0)$ & $0(0.0)$ \\
\hline Conduction disorder & $1(0.2)$ & $5(0.03)$ \\
\hline
\end{tabular}

* Severe CHF defined by presence of a diagnosis for decompensated $\mathrm{CH}$ or by any CHF diagnosis followed by a prescription of a loop diuretic within 3 months.

the focus of exposure was on current use, we defined as "no use" the absence of prescriptions of a given drug during the year prior to the index date. Patients with prescriptions for a given drug during the year before the index date, but not current users, were classified as past users.

\section{Covariates}

Risk factors for the outcome were identified from patients' records in the GPRD. In particular, we identified depression related factors (alcohol abuse, suicide attempt, depression severity), ${ }^{24}$ cardiovascular risk factors (obesity, smoking, diabetes, left ventricular hypertrophy, hyperlipidaemia, hypertension, rheumatoid arthritis), cardiovascular diagnoses (acute myocardial infarction, coronary artery bypass graft and percutaneous coronary artery procedures, intraventricular conduction delay, supraventricular arrhythmia, atrial arrhythmia, coronary artery disease, angina, congestive heart failure, ischaemic stroke, transient ischaemic attack, peripheral vascular disease), other conditions that have been associated with an increased risk for sudden cardiac death (epilepsy and schizophrenia), and conditions or use of drugs that could prolong the QT interval, including hypokalaemia, hypomagnesaemia, and conduction disorders. ${ }^{2526} \mathrm{We}$ also measured use of certain classes of drug including non-steroidal anti-inflammatory drugs (NSAIDs), antipsychotics, benzodiazepines, mood stabilisers, antiarrhythmic agents, lipid regulating drugs, and loop diuretics. Lastly, we identified individuals who had switched antidepressants between the time of cohort entry and the index date. Except for obesity, which we defined as a body mass index (BMI) of more than 30, covariates were ascertained from diagnoses, lifestyle factors, or prescriptions as they appeared in the medical record.

\section{Data analysis}

We used conditional logistic regression to compute the odds ratio of sudden cardiac death or near death, and its $95 \%$ confidence interval, associated with current exclusive use of venlafaxine compared with current exclusive use of each of the three other study drugs, as well as compared with the three drugs as one group. In a nested case-control study such as ours, the odds ratio provides an unbiased estimate of the rate ratio in the cohort. ${ }^{27}$

Because the number of controls matched to each case was variable and to allow unbiased comparisons between cases and controls, descriptive statistics for the characteristics of the controls were weighted by the inverse of the number of controls in each matched set, a procedure equivalent to standardising the number of controls to one control per case. After tabulating the data, we performed crude regression analyses. All analyses were then adjusted for comorbidity and other covariates measured 366-730 days before the index date, to avoid adjusting for factors affected by exposure during the year prior to the index date. We also assessed the effect of duration of exposure in patients currently exposed to the study drugs.

We performed several sensitivity analyses. We repeated the main analyses after restricting our outcome to the first two sources of cases (non-fatal acute ventricular tachyarrhythmia as well as sudden deaths due to any cardiac pathology). We repeated the main analyses adding a lag of 15 days to the end of the prescription duration, to allow for possible late exposures beyond the prescription duration. We repeated the main analyses using two alternative time periods in which covariates were measured. We first adjusted for comorbidities measured in the year beginning 415 days before the index date, instead of the year beginning 730 days before the index date, and then adjusted only for covariates ascertained prior to cohort entry, to address any concerns about adjusting for factors that were actually drug effects. We also restricted the covariates to those that affected the odds ratio by $10 \%$ or more. Finally, to assess effect modification, the estimates were stratified by the indication for the antidepressant, by the presence or absence of a diagnosis of myocardial infarction before the index date, and by the occurrence of switching among antidepressant classes 
Table $3 \mid$ Comorbidity and drug use of cases and controls in the year before the year before index date. Data are frequencies (percentages) unless otherwise stated. Percentages cannot be calculated directly from the corresponding frequencies as they are weighted by the number of controls matched to each case

\begin{tabular}{|c|c|c|}
\hline & $\begin{array}{l}\text { Cases } \\
(n=568)\end{array}$ & $\begin{array}{c}\text { Controls } \\
(n=14812)\end{array}$ \\
\hline \multicolumn{3}{|c|}{ Non-cardiovascular comorbidity } \\
\hline Rheumatoid arthritis & $4(0.7)$ & $72(0.5)$ \\
\hline Epilepsy & $4(0.7)$ & $68(0.5)$ \\
\hline Schizophrenia & $3(0.5)$ & $10(0.1)$ \\
\hline \multicolumn{3}{|l|}{ Drug use } \\
\hline NSAIDs & $273(48.1)$ & $6443(43.7)$ \\
\hline Antipsychotics & $83(14.6)$ & $1840(12.9)$ \\
\hline Benzodiazepines & $181(31.9)$ & $3582(25.4)$ \\
\hline Mood stabilisers & $26(4.6)$ & $573(3.8)$ \\
\hline Antiarrhythmics & $17(3.0)$ & $270(1.8)$ \\
\hline Lipid regulating drugs & $65(11.4)$ & $1171(7.8)$ \\
\hline Loop diuretics & $151(26.6)$ & $1860(13.4)$ \\
\hline \multicolumn{3}{|c|}{ Drugs with potential effect on QT prolongation* } \\
\hline Strong evidence & $172(30.3)$ & $3494(23.6)$ \\
\hline Soft evidence & $87(15.3)$ & $1820(12.3)$ \\
\hline
\end{tabular}

NSAID=non-steroidal anti-inflammatory drugs.

*Prescribed drugs associated with QT prolongation were stratified into two groups based on the strength of evidence supporting the drug's propensity to prolong the $\mathrm{QT}$ interval. ${ }^{22,23}$

as measured by a change of the current antidepressant from the cohort entry defining agent.

We used SAS v9.1. (Cary, NC, USA) statistical software for all analyses. The study protocol was approved by the Independent Scientific Advisory Committee for GPRD research, and this report includes all relevant STROBE elements.

\section{RESULTS}

The initial cohort included 269084 individuals with an incident prescription of one of the study drugs after January 1995 and with at least a year of data prior to that prescription. We then excluded 2426 patients because they were younger than 18 years or older than 90 at the date of the incident prescription; 58464 patients without a diagnosis of depression or anxiety before or concurrent with the incident prescription; 473 patients with congenital conduction disorder, cardiomyopathy, or history of acute ventricular tachyarrhythmia; 284 patients with cardioversion, cardiac arrest with resuscitation, or implantation of an internal cardiac defibrillator prior to cohort entry; and 53 patients with incomplete follow-up information. Thus, the final study cohort consisted of 207384 first time users of venlafaxine $(n=19268)$, citalopram $(\mathrm{n}=53300)$, fluoxetine $(\mathrm{n}=90924)$, or dosulepin $(\mathrm{n}=$ 43892 ). Over the study period, 17783 patients who entered the study taking one of the comparator antidepressants were subsequently prescribed venlafaxine, bringing the total number of individuals exposed to venlafaxine to 35051 .

The mean age at cohort entry was 46 years, with a mean of 7.8 years of available data in the practice between the time it was deemed up to standard for data collection purposes and cohort entry and a mean follow-up of 3.3 years. A third of cohort members were men. The diagnosis associated with antidepressant use was depression in $84 \%$ and anxiety in $16 \%$. Almost half of the patients had never been prescribed any other antidepressant before the qualifying prescription. Compared with patients initiating fluoxetine, citalopram, or dosulepin, patients initiating venlafaxine were more likely to have previously used tricyclics, SSRIs, monamine oxidase inhibitors, or other antidepressants (supplemental table 1, see webextras). They also were more likely to have had more severe depression or attempted suicide (supplemental table 2). Patients initiating venlafaxine were no different in terms of their cardiovascular comorbidities at baseline but had greater use of antipsychotic drugs, benzodiazepines, and mood stabilisers, as well as somewhat higher use of drugs that may prolong the QT interval (supplemental tables 3-4).

The figure 1 describes the algorithm to identify the three sets of out of hospital cases. Using this algorithm, we identified 568 cases, including 27 acute ventricular tachyarrhythmias, 236 sudden cardiac deaths, and 305 out of hospital cardiac ischaemic deaths. The initial concordance rate regarding case status between the two authors after their manual review of all potential acute ventricular tachyarrhythmia and of the random set of 50 potential sudden death cases was 73\%, and all disagreements were resolved after discussion.

The overall rate of sudden cardiac death or near death was 8.21 per 10000 person-years. The 568 cases were matched to 14812 controls, with a mean age of 73 years at the index date. Cases were more likely to be smokers, to abuse alcohol, and to have attempted suicide, despite no major differences in the severity of depression (table 1). In the year prior to the year before the index date, cases generally had a higher prevalence of cardiovascular related comorbidity, particularly diabetes, acute myocardial infarction, congestive heart failure, and atrial arrhythmia (table 2). Cases also had a higher prevalence of rheumatoid arthritis, epilepsy, and schizophrenia, as well as use of NSAIDs, benzodiazepines, lipid regulating agents, loop diuretics, and drugs that may prolong the QT interval (table 3). Although all patients were exposed to one of the study drugs at cohort entry, over time many did not continue on these treatments, as would be expected in clinical practice. On the index date only a subset of cases $(n=155)$ and controls $(n=3916)$ were currently exposed to a study drug.

Current venlafaxine use was not associated with an increased risk of sudden cardiac death or near death compared with current use of fluoxetine, citalopram, and dosulepin, with all point estimates of the adjusted odds ratio less than 1 (table 4 , model 1). In an alternative model that included only covariates that were measured before cohort entry, results were essentially the same (table 4, model 2). Stratified analyses did not show any significant variations in risk by duration of 
Table 4 |Crude and adjusted odds ratios of sudden cardiac death or near death associated with current use of venlafaxine relative to current fluoxetine, citalopram, and dosulepin use

\begin{tabular}{|c|c|c|c|c|c|}
\hline & $\begin{array}{l}\text { Cases } \\
(n=568)\end{array}$ & $\begin{array}{c}\text { Controls } \\
(n=14812)\end{array}$ & Crude odds ratio & $\begin{array}{l}\text { Adjusted odds ratio } \\
\text { Model } 1 *(95 \% \mathrm{Cl})\end{array}$ & $\begin{array}{l}\text { Adjusted odds ratio } \\
\text { Model } 2 \dagger(95 \% \mathrm{Cl})\end{array}$ \\
\hline Venlafaxine & $18(3.2)$ & $544(3.7)$ & & & \\
\hline Versus fluoxetine & $63(11.1)$ & $1281(8.6)$ & 0.65 & $0.66(0.38$ to 1.14$)$ & $0.66(0.38$ to 1.14$)$ \\
\hline Versus citalopram & $39(6.9)$ & $1079(7.3)$ & 0.90 & $0.89(0.50$ to 1.60$)$ & $0.88(0.49$ to 1.56$)$ \\
\hline Versus dosulepin & $35(6.2)$ & $1012(6.8)$ & 0.99 & 0.83 (0.46 to 1.52$)$ & 0.86 (0.48 to 1.56$)$. \\
\hline Versus any of the three & $137(24.1)$ & $3372(22.8)$ & 0.81 & 0.77 (0.46 to 1.30$)$ & 0.78 (0.47 to 1.29$)$ \\
\hline
\end{tabular}

*Model 1 adjusted for BMI $\geq 30$, smoking status, alcohol abuse, depression severity, suicide attempt, diabetes, coronary artery bypass graft and endoscopic cardiac procedures, supraventricular arrhythmias, left ventricular hypertrophy, coronary artery disease and angina, congestive heart failure, severe congestive heart failure, ischaemic stroke, transient ischaemic attack, peripheral vascular disease, hyperlipidaemia, hypertension, hypokalaemia, rheumatoid arthritis, epilepsy, schizophrenia, use of antipsychotics, benzodiazepine, mood stabilisers, antiarrhythmics, drugs with some evidence of prolonging QT, drugs with stronger evidence of prolonging QT, lipid regulating drugs, loop diuretics-all ascertained in the year prior to the year before index date and acute myocardial infarction in the year before index date.

†Adjusted for all factors listed for model 1 as ascertained before cohort entry. Models 1 and 2 included non-users and past users, in addition to current users. Because the contrasts here are limited to current users, drug specific counts of cases and controls do not add up to column totals.

use of any of the four antidepressants used to define the cohort (table 5).

Sensitivity analyses restricting our outcome to acute ventricular tachyarrhythmia and sudden cardiac death did not change the results, nor did adding a lag of 15 days to the end of the prescription duration (supplemental tables 5-6). Varying the time window to define covariates or adjusting only for covariates that changed the odds ratio by at least $10 \%$ also did not materially affect the results (supplemental table 7). Finally, results were similar in models restricted to patients with a diagnosis of depression as indication for the drugs or among patients with no diagnosis of acute myocardial infarction before the index date (supplemental tables 89). Switching from the antidepressant used at cohort entry to the one at index date was not associated with an increased risk of our composite outcome (odds ratio $0.83 ; 95 \%$ confidence interval 0.52 to 1.32 ). In an analysis of switching stratified by each study drug, the results did not change although the numbers were small (supplemental tables 10).

\section{DISCUSSION}

In this large population based cohort study of patients treated for depression or anxiety, we found no evidence that venlafaxine use was associated with a higher risk of out of hospital haemodynamically significant acute ventricular tachyarrhythmia or sudden cardiac death compared with the risk observed in fluoxetine, citalopram, or dosulepin users. While only 18 cases of sudden cardiac death or near death occurred in patients currently exposed to venlafaxine, the study allows us reasonably to exclude odds ratios higher than 1.14 compared with current fluoxetine use, 1.60 compared with current citalopram use, 1.52 compared with current dosulepin use, and 1.30 compared with current use of any of the three comparators, based on the upper bound of the $95 \%$ confidence interval for each of the four comparisons. We also found no compelling evidence that venlafaxine was preferentially prescribed to patients with lower risk for cardiac events, consistent with the observation that adjustment for potential confounders had minimal effect on results.

\section{Comparison with other studies}

The motivation for this investigation arose from three recent observational studies that reported a higher rate of fatal antidepressant overdose with venlafaxine use compared with SSRIs. ${ }^{1-3}$ While one interpretation of these findings is that venlafaxine is relatively more toxic in overdose, patient factors rather than differential drug effects could also explain the phenomenon. None of these studies adjusted for potential confounding factors, and in fact a UK based utilisation study demonstrated that venlafaxine was preferentially prescribed to patients with a higher prevalence of risk factors for suicide. ${ }^{28}$ The cause of venlafaxine overdose deaths has not been well characterised, although a cardiotoxic mechanism, mediated through malignant ventricular tachyarrhythmias, has been suggested. The current study assessed the risk of malignant ventricular tachyarrhythmias and sudden cardiac death among patients receiving venlafaxine and comparator antidepressants at pharmacological doses, and found no such association.

While some studies compare current use of a drug to non-use to assess drug risk, we chose to compare current use of one drug to another drug. We believe that this approach is clinically sensible and minimises confounding by indication. We designed the study to address the following question a clinician might ask him or herself: "Given that I have chosen to treat my patient with an antidepressant, will the choice of venlafaxine instead of other antidepressants increase my patient's risk of unexpected cardiac death or life threatening ventricular tachyarrhythmia?" Alternatively, one could compare current use to distant past use, but such an approach could be vulnerable to confounding by indication. Several studies have suggested that depressed individuals are at increased risk for cardiac mortality compared with non-depressed individuals. ${ }^{29-32}$ Less clear is whether depression associated risk of adverse cardiovascular outcomes attenuates when depression abates. If this is the case, an 
Table $5 \mid$ Crude and adjusted rate ratios of sudden cardiac death or near death associated with current use of venlafaxine, fluoxetine, citalopram, and dosulepin, comparing longer with shorter duration of current use. Data are number (percentage) unless otherwise specified

\begin{tabular}{|c|c|c|c|c|}
\hline & Cases $(n=568)$ & Controls ( $n=14812$ ) & Crude odds ratio & Adjusted odds ratio* $(95 \% \mathrm{Cl})$ \\
\hline \multicolumn{5}{|l|}{ Venlafaxine } \\
\hline$<90$ days & $2(0.4)$ & $70(0.5)$ & 1.00 & $1.32(0.26$ to 6.70$)$ \\
\hline$\geq 90$ days & $16(2.8)$ & $474(3.2)$ & 1.00 (reference) & 1.00 (reference) \\
\hline \multicolumn{5}{|l|}{ Fluoxetine } \\
\hline$<90$ days & $8(1.4)$ & $261(1.8)$ & 0.61 & $0.70(0.25$ to 1.93$)$ \\
\hline$\geq 90$ days & $55(9.7)$ & $1020(6.9)$ & 1.00 (reference) & 1.00 (reference) \\
\hline \multicolumn{5}{|l|}{ Citalopram } \\
\hline$<90$ days & $8(1.4)$ & $153(1.0)$ & 1.94 & 1.81 (0.63 to 5.21$)$ \\
\hline$\geq 90$ days & $31(5.5)$ & $926(6.3)$ & 1.00 (reference) & 1.00 (reference) \\
\hline \multicolumn{5}{|l|}{ Dosulepin } \\
\hline$<90$ days & $8(1.4)$ & $207(1.4)$ & 1.27 & $1.44(0.50$ to 4.16$)$ \\
\hline$\geq 90$ days & $27(4.8)$ & $805(5.4)$ & 1.00 (reference) & 1.00 (reference) \\
\hline \multicolumn{5}{|c|}{$\begin{array}{l}\text { *Adjusted for BMI } \geq 30 \text {, smoking status, alcohol abuse, depression severity, suicide attempt, diabetes, coronary artery bypass graft and endoscopic } \\
\text { coronary artery procedures, supraventricular arrhythmias, left ventricular hypertrophy, coronary artery disease and angina, congestive heart failure, } \\
\text { severe congestive heart failure, ischaemic stroke, transient ischaemic attack, peripheral vascular disease, hyperlipidaemia, hypertension, } \\
\text { hypokalaemia, rheumatoid arthritis, epilepsy, schizophrenia, use of antipsychotics, benzodiazepine, mood stabilisers, antiarrhythmics, drugs with } \\
\text { some evidence of prolonging QT, drugs with stronger evidence of prolonging QT, lipid regulating drugs, loop diuretics all in the year prior to the year } \\
\text { before index date and acute myocardial infarction in the year prior index date. Because the contrasts here are limited to current users, drug specific } \\
\text { counts of cases and controls do not sum to column totals. }\end{array}$} \\
\hline
\end{tabular}

association emerging from a comparison of current antidepressant use with distant past use could easily be a consequence of confounding by time dependent patient factors associated with depression itself. Thus, we selected three other antidepressant agents to define the comparison group, namely fluoxetine, citalopram, and dosulepin, as in a previous study of suicide risk associated with venlafaxine that used the same dataset. ${ }^{19}$ Fluoxetine, an SSRI, and dosulepin, a tricyclic antidepressant, were selected because they were the most commonly prescribed drugs within their respective classes during the study period. As citalopram and venlafaxine were introduced in the same year, we assumed that doctors would preferentially prescribe both agents to patients who were unresponsive to previously available therapies. ${ }^{5}$ To the degree that severity of depression could increase risk of cardiac events, ${ }^{29}$ we believed that selecting citalopram as a comparator would minimise confounding by disease severity.

Like other antidepressants of the tricyclic class, dosulepin in the overdose setting can produce malignant arrhythmias, some fatal. ${ }^{3334}$ We identified only one population based study that evaluated the risk of sudden cardiac death involving tricyclic antidepressants at pharmacologic doses. ${ }^{35}$ This study found that, compared with non-use of antidepressants, tricyclic antidepressants were not associated with an increased risk of sudden cardiac death at doses of less than $100 \mathrm{mg}$ per day of amitriptyline equivalents. An increased risk, however, was observed at higher doses. Our GPRD study did not suggest an excess risk of sudden cardiac death or near death associated with dosulepin use. We suspect that the relatively infrequent use at higher doses may explain this finding. Among patients currently exposed to dosulepin on the index date for whom a daily dose could be measured, $87.6 \%$
(840/959) were prescribed less than $100 \mathrm{mg}$ /day (we assume that amitriptyline and dosulepin are equivalent on a $\mathrm{mg} / \mathrm{mg}$ basis). ${ }^{36}$

\section{Limitations of study}

Our study has some limitations, most of which involve the level of detail in the GPRD medical history and the challenge of determining the precise sequence of events in patients who died out of hospital. Firstly, we could not apply standardised definitions of sudden cardiac death such as those used in large clinical trials, since we lacked access to complete medical records and the ability to interview family members of deceased people. However, our definition of sudden cardiac death was largely consistent with the one used in a recent study of antipsychotic drug use and sudden cardiac death, although ours also included cases of life threatening but non-fatal ventricular arrhythmias. ${ }^{37}$ Secondly, we cannot be certain that all of the fatal cases included in this study had a malignant ventricular tachyarrhythmia as the final cardiac event before death. In large part, this limitation is a consequence of most fatal cases both not being on an electrocardiograph at the time of haemodynamic collapse and not subsequently undergoing autopsy. Furthermore, we did not have access to medical records detailing the cardiac rhythm before death in the few cases whose arrest was witnessed and managed by paramedics or doctors. Community studies suggest that some patients who die shortly after haemodynamic collapse from a cardiac cause may not go through a phase of ventricular tachyarrhythmia or fibrillation before death. ${ }^{3839}$ Nevertheless, we suspect that among cases who did not have a malignant tachyarrhythmia, most died from some cardiac cause. To the degree that this occurred, it is reassuring that venlafaxine was not 


\section{WHAT IS ALREADY KNOWN ON THIS TOPIC}

In recent reports from the UK, the antidepressant venlafaxine was associated with an increased rate of fatal overdose compared with several other SSRIS

The finding might be due to patient factors, as venlafaxine has been systematically prescribed to sicker patients who are at higher risk for suicide, or to inherent toxicity of venlafaxine, possibly because of a pro-arrhythmic mechanism

Whether use of venlafaxine at therapeutic doses is associated with an increased risk of sudden cardiac death or life threatening arrhythmia has not been studied

\section{WHAT THIS STUDY ADDS}

Using data from the General Practice Research Database, this observational study of more than 200000 patients treated for depression or anxiety found no excess risk of sudden death or near death associated with use of venlafaxine compared with other commonly used antidepressants

\section{Conclusion}

In this large UK population based study in patients with depression or anxiety, venlafaxine was not associated with any excess risk of malignant ventricular tachyarrhythmia or sudden cardiac death when compared with fluoxetine, dosulepin, or citalopram.

Contributors: CM, DM, SS were responsible for the conception and design of the study. SD was responsible for the statistical analysis. CM and TA adjudicated cases of non-fatal ventricular arrhythmias. All authors contributed to the interpretation of results and manuscript preparation and granted final approval of this report. CM and SS are guarantors. Funding: This study was sponsored by Wyeth, which produces and markets venlafaxine. The contract for this research specified that the noncompany authors had ultimate control over all aspects of the study, including control over publication. During the course of the study, however, any differences about the presentation or interpretation of findings that arose between the company author and external investigators were resolved through honest scientific debate. All authors had access to the statistical reports and tables supporting the publication. Competing interests: DM is a employee of Wyeth and owns company stock options. SS has participated in advisory board meetings and conferences, participated as a speaker in scientific meetings by various companies (AstraZeneca, Boehringer Ingelheim, Glaxo SmithKline, Pfizer and Sepracor), and received research grants from AstraZeneca, Wyeth, and GlaxoSmithKline. TA, SD, and CM have nothing to declare.

Ethical approval: The study protocol was approved by the Independent Scientific Advisory Committee for GPRD studies (Protocol 06_054). Data sharing: Read/OXMIS code lists used to identify outcomes are available from the corresponding author at samy.suissa@mcgill.ca.

1 Buckley NA, McManus PR. Fatal toxicity of serotoninergic and other antidepressant drugs: analysis of United Kingdom mortality data. BMJ 2002;325:1332-3.

2 Morgan O, Griffiths C, Baker A, Majeed A. Fatal toxicity of antidepressants in England and Wales, 1993-2002. Health Stat $Q$ 2004; $23: 18-24$.

3 Cheeta S, Schifano F, Oyefeso A, Webb L, Ghodse AH. Antidepressant-related deaths and antidepressant prescriptions in England and Wales, 1998-2000. Br J Psychiatry 2004;184:41-7.

4 Heerdink ER, Hugenholtz GW, Meijer WE, Egberts AC. Channelling new antidepressants to problem patients may be factor in fatal toxicity. BM/ 2003;326:600.

5 Egberts AC, Lenderink AW, de Koning FH, Leufkens HG. Channeling of three newly introduced antidepressants to patients not responding satisfactorily to previous treatment. J Clin Psychopharmacol 1997;17:149-55.

6 Khalifa M, Daleau P, Turgeon J. Mechanism of sodium channel block by venlafaxine in guinea pig ventricular myocytes. J Pharmacol Exp Ther 1999;291:280-4.

7 Whyte IM, Dawson AH, Buckley NA. Relative toxicity of venlafaxine and selective serotonin reuptake inhibitors in overdose compared to tricyclic antidepressants. QIM 2003;96:369-74.

8 Product Monograph Effexor XR Capsules (venlafaxine hydrochloride), extended release capsules $(37.5,75,150 \mathrm{mg})$. www.wyeth.ca/en/ products/Product\%20Monographs\%20PDFs/Effexor\%20PRODUCT \%20MONOGRAPH\%2010Jan07.pdf.

9 Electronic Medicines Compendium (eMC). Efexor-summary of product characteristics. 2008. http://emc.medicines.org.uk/emc/ assets/c/html/DisplayDoc.asp?DocumentID=2209.

10 Johnson EM, Whyte E, Mulsant BH, Pollock BG, Weber E, Begley AE, et al. Cardiovascular changes associated with venlafaxine in the treatment of late-life depression. Am J Geriatr Psychiatry 2006;14:796-802.

11 Colbridge MG, Volans GN. Venlafaxine in overdose-experience of the National Poisons Information Service (London centre). J Toxicol Clin Toxicol 1999;37:383.

12 Kelly CA, Dhaun N, Laing WJ, Strachan FE, Good AM, Bateman DN. Comparative toxicity of citalopram and the newer antidepressants after overdose. J Toxicol Clin Toxicol 2004;42:67-71.

13 Thase ME. Effects of venlafaxine on blood pressure: a meta-analysis of original data from 3744 depressed patients. J Clin Psychiatry 1998;59:502-8.

14 Rudolph RL, Fabre LF, Feighner JP, Rickels K, Entsuah R, Derivan AT. A randomized, placebo-controlled, dose-response trial of venlafaxine hydrochloride in the treatment of major depression. J Clin Psychiatry 1998;59:116-22.

15 Medicines and Healthcare Products Regulatory Agency. Experts complete review on safety of antidepressants. 2005. www.mhra.gov. uk/NewsCentre/Pressreleases/CON002060. 
16 Medicines and Healthcare Products Regulatory Agency. Updated summary of the Committee on Safety of Medicines meeting held on 25 November 2004. 2007. www.mhra.gov.uk/home/groups/l-cs-el/ documents/committeedocument/con003208.pdf.

17 Medicines and Healthcare Products Regulatory Agency. Venlafaxine (Efexor): summary of basis for regulatory position. 2006. www.mhra. gov.uk/Safetyinformation/Safetywarningsalertsandrecalls/ Safetywarningsandmessagesformedicines/CON2023846.

18 Wood L, Martinez C. The general practice research database: role in pharmacovigilance. Drug Saf 2004;27:871-81.

19 Rubino A, Roskell N, Tennis P, Mines D, Weich S, Andrews E. Risk of suicide during treatment with venlafaxine, citalopram, fluoxetine, and dothiepin: retrospective cohort study. BMJ 2007;334:242.

20 General Practice Research Database. Facts and figures. 2009. www. gprd.com/gprd/factsandfigures.asp.

21 Essebag V, Genest J Jr, Suissa S, Pilote L. The nested case-control study in cardiology. Am Heart / 2003;146:581-90.

22 Suissa S. Novel approaches to pharmacoepidemiology study design and statistical analysis. In: Strom BL, ed. Pharmacoepidemiology. John Wiley \& Sons, 2005:811-29.

23 Zipes DP, Wellens HJ. Sudden cardiac death. Circulation 1998;98:2334-51.

24 Martinez C, Rietbrock S, Wise L, Ashby D, Chick J, Moseley J, et al. Antidepressant treatment and the risk of fatal and non-fatal self harm in first episode depression: nested case-control study. $B M$ J 2005;330:389-93.

25 Arizona Center for Education and Research on Therapeutics. QT drug lists by risk groups: drugs that prolong the QT interval and/or induce torsades de pointes ventricular arrhythmia. www.azcert.org/ medical-pros/drug-lists/drug-lists.cfm.

26 LaPointe NM, Curtis LH, Chan KA, Kramer JM, Lafata JE, Gurwitz JH, et al. Frequency of high-risk use of QT-prolonging medications. Pharmacoepidemiol Drug Saf 2006;15:361-8.

27 Rothman KJ, Greenland S, Lash TL. Case-control studies. In Rothman KJ, Greenland S, Lash T, eds. Modern epidemiology. 3rd ed. Lippincott Williams-Wilkins, 2008:111-27.

28 Mines D, Hill D, Yu H, Novelli L. Prevalence of risk factors for suicide in patients prescribed venlafaxine, fluoxetine, and citalopram. Pharmacoepidemiol Drug Saf 2005;14:367-72.

29 Rowan PJ, Haas D, Campbell JA, Maclean DR, Davidson KW. Depressive symptoms have an independent, gradient risk fo coronary heart disease incidence in a random, population-based sample. Ann Epidemiol 2005;15:316-20.

30 Ariyo AA, Haan M, Tangen CM, Rutledge JC, Cushman M, Dobs A, et al: Cardiovascular Health Study Collaborative Research Group. Depressive symptoms and risks of coronary heart disease and mortality in elderly Americans. Circulation 2000;102:1773-9.

31 Anda R, Williamson D, Jones D, Macera C, Eaker E, Glassman A, et al. Depressed affect, hopelessness, and the risk of ischemic heart disease in a cohort of US adults. Epidemiology 1993;4:285-94.

32 Frasure-Smith N, Lespérance F. Depression and anxiety as predictors of 2-year cardiac events in patients with stable coronary artery disease. Arch Gen Psychiatry 2008;65:62-71.
33 Buckley NA, Dawson AH, Whyte IM, Henry DA. Greater toxicity in overdose of dothiepin than of other tricyclic antidepressants. Lancet 1994;343:159-62.

34 Chan CY, Waring WS, Chan CYY, Waring WS. Images in cardiovascular medicine. Tricyclic cardiotoxicity treated with sodium bicarbonate. Circulation 2007;115:e63-4.

35 Ray WA, Meredith S, Thapa PB, Hall K, Murray KT. Cyclic antidepressants and the risk of sudden cardiac death. Clin Pharmacol Ther 2004;75:234-41.

36 British Medical Association and the Royal Pharmaceutical Society of Great Britain. British National Formulary: 58 (September 2009). Pharmaceutical Press, 2009.

37 Ray WA, Chung CP, Murray KT, Hall K, Stein CM. Atypical antipsychotic drugs and the risk of sudden cardiac death. N EnglJ Med 2009;360:225-35.

38 Herlitz J, Engdahl J, Svensson L, Young M, Angquist KA, Holmberg S. Changes in demographic factors and mortality after out-of-hospital cardiac arrest in Sweden. Coron Artery Dis 2005;16:51-7.

39 De Maio VJ, Stiell IG, Wells GA, Spaite DW: OPALS study group. Cardiac arrest witnessed by emergency medical services personnel: descriptive epidemiology, prodromal symptoms, and predictors of survival. Ann Emerg Med 2000;35:138-46.

40 Centers for Disease Control and Prevention (CDC). State-specific mortality from sudden cardiac death—United States, 1999. MMWR Morb Mortal Wkly Rep 2002;51:123-6.

41 Pratt CM, Hertz RP, Ellis BE, Crowell SP, Louv W, Moyé L. Risk of developing life-threatening ventricular arrhythmia associated with tefenadine in comparison with over-the-counter antihistamines, ibuprofen and clemastine. Am J Cardiol 1994;73:346-52.

42 Hennessy S, Bilker WB, Knauss JS, Margolis DJ, Kimmel SE, Reynolds RF, et al. Cardiac arrest and ventricular arrhythmia in patients taking antipsychotic drugs: cohort study using administrative data. BMJ 2002;325:1070.

43 Enger C, Cali C, Walker AM. Serious ventricular arrhythmias among users of cisapride and other QT-prolonging agents in the United States. Pharmacoepidemiol Drug Saf 2002;11:477-86.

44 Walker AM, Szneke P, Weatherby LB, Dicker LW, Lanza LL, Loughlin JE, et al. The risk of serious cardiac arrhythmias among cisapride users in the United Kingdom and Canada. Am J Med 1999;107:356-62.

45 Hennessy S, Leonard CE, Palumbo CM, Bilker WB, Newcomb C, Kimmel SE. Diagnostic codes for sudden cardiac death and ventricular arrhythmia functioned poorly to identify outpatient events in EPIC's General Practice Research Database. Pharmacoepidemiol Drug Saf 2008;17:1131-6.

46 Nemeroff CB, Entsuah R, Benattia I, Demitrack M, Sloan DM, Thase ME. Comprehensive analysis of remission (COMPARE) with venlafaxine versus SSRIs. Biol Psychiatry 2008;63:424-34.

Accepted: 25 November 2009 